\title{
Contextual influences of dimension, speed, and direction of motion on subjective time perception
}

\author{
Spencer R. Kline • Catherine L. Reed
}

Published online: 14 September 2012

(C) Psychonomic Society, Inc. 2012

\begin{abstract}
Research has indicated that the direction of motion and the speed of motion can influence the subjective estimates of temporal duration of two-dimensional (2-D) stimuli expanding and contracting within the picture plane. In this study, we investigated whether the contextual cues of stimulus/movement-plane dimensionality (2-D stimuli with implied movement in the picture plane or depth-rendered "3-D" stimuli with implied movement in the depth plane) influence and interact with speed and implied movement direction during interval estimation. Participants viewed a series of standard stimulus durations followed by a test stimulus duration and determined whether the test and standard durations differed. The results indicated that moving stimuli were overestimated relative to stationary stimuli, regardless of the direction of motion or dimensionality. Also, faster-moving stimuli were overestimated relative to slower-moving stimuli. Importantly, an interaction between movement direction and dimensional cues indicated that the loom/recede distinction occurs for 2-D but not for 3-D stimuli. It is possible that the loom/recede distinction for the 2-D condition may be an artifact arising from reduced or from a lack of perceived motion in 2-D "recede" conditions, rather than a specific overestimation for looming stimuli.
\end{abstract}

Keywords Time perception - Subjective interval estimation · Implied motion · Visual illusion · Looming · Receding

In this study, we investigated whether the contextual cues of movement direction, speed, and dimensionality interact to

\section{S. R. Kline $\cdot$ C. L. Reed $(\bowtie)$}

Department of Psychology, Claremont McKenna College,

850 Columbia Avenue,

Claremont, CA 81711, USA

e-mail: clreed@cmc.edu influence our perception of temporal intervals. Time perception depends on a variety of different situational factors (Angrilli, Cherubini, \& Manfredini, 1997; Droit-Volet \& Meck, 2007; Lewis \& Miall, 2003; Wearden \& PentonVoak, 1995; Zakay \& Block, 2004). The physical characteristics of stimuli (e.g., the intensity of light or sounds; Fraisse, 1984), emotional valence (Droit-Volet \& Gil, 2009), and attentional load (Block \& Zakay, 1997), as well as pharmacological manipulations in animals (Buhusi \& Meck, 2002; Meck, 1996; Penney, Holder, \& Meck, 1996), influence the estimation of duration. Furthermore, manipulations of attention, arousal, and cognitive-processing demand have been shown to also affect perceived interval duration. Highly arousing stimuli are often perceived as being longer than neutral stimuli (Burle \& Casini, 2001; Droit-Volet \& Wearden 2002; Ortega, Lopez, \& Church, 2009), and estimates made while performing a concurrent task are shorter than estimates made in the absence of such extra cognitive load (Brown, 1997; Droit-Volet, 2003). Also, the overestimation of the duration of a deviant stimulus within a stream of standard events has been attributed to an increase in the attention directed to the deviant stimulus (New \& Scholl, 2009; Tse, Intriligator, Rivest, \& Cavanagh, 2004; van Wassenhove, Buonomano, Shimojo, \& Shams, 2008); to coding efficiency, in which the encoding of a deviant stimulus dilates experienced duration (Eagleman \& Pariyadath, 2009); and to the intrinsic dynamics of the stimulus (Kanai, Paffen, Hogendoorn, \& Verstraten, 2006; New \& Scholl, 2009).

Movement provides one of the most robust contextual influences on perceived interval duration (Brown, 1995; Roelofs \& Zeeman, 1951-1952; Tayama, Nakamura, \& Aiba, 1987). Brown (1995) found that when participants were asked to reproduce the durations of moving and stationary stimuli, the perceived duration of moving stimuli was longer than that of stationary stimuli. Furthermore, increasing the speed of movement led to further interval 
dilation: Fast-moving stimuli elicited longer reproductions than did slow-moving stimuli. For instance, Mate, Pires, Campoy, and Estaun (2009) used a constant-stimulus method and compared slow- and fast-moving stimuli with stationary stimuli. They found that not only did moving stimuli produce greater temporal overestimation than did stationary stimuli, but also that faster stimuli produced greater overestimations than did slower stimuli.

Perceived interval duration is influenced not only by movement per se, but also by the direction of motion. Looming signals of two-dimensional (2-D) stimuli (i.e., discs that expand in diameter) are associated with subjective time dilation, whereas receding signals (i.e., discs that contract in size) are not (New \& Scholl, 2009; van Wassenhove et al., 2008; Wittmann, van Wassenhove, Craig, \& Paulus, 2010). For example, Wittmann et al. presented participants with trials consisting of three successive stationary stimuli at a standard duration, followed by a test stimulus that appeared to loom, recede, or remain stationary, followed by a standard stimulus. Participants then indicated whether the duration of the test stimulus was longer or shorter than the duration of the standard stimuli. The participants indicated that the test stimuli were "longer" a higher percentage of the time when the stimuli loomed than when they receded or remained stationary. No difference emerged between the percentages of "longer" responses for test stimuli that receded or remained stationary. This occurred even though the degrees of "change" associated with both looming and receding stimuli were identical. These observations suggest that looming stimuli are particularly salient events (Yantis \& Egeth, 1999) and that they draw attention as compared to other stimuli (Franconeri, Hollingworth, \& Simons, 2005). Looming signals are an intrinsic threat cue for the organism (Abrams \& Christ, 2005; Droit-Volet \& Gil, 2009; Schiff et al., 1962) and may constitute a natural self-referential stimulus (Northoff, Heinzel, de Greck, Bermpohl, Dobrowolny, \& Panksepp, 2006).

Several models of interval perception have been proposed to account for motion speed and direction findings. The memory trace model (Ornstein, 1969) or contextual change model (CCM) proposes that contextual changes (e.g., alternation of an individual's emotional state or of stimulus complexity, changes occurring in a stimulus's presentation, or other variations that break an interval into discrete segments) lead to a dilation of perceived interval duration, because the changes create a higher number of discrete memory traces available for retrieval (Block, 1982; Zakay \& Block, 2004). The perceived duration of moving versus stationary stimuli may be overestimated because moving stimuli lead to more changes and more discrete memory traces (Poynter, 1989). Likewise, faster stimuli may be overestimated more than slower stimuli because they move a greater distance over a comparable interval than do slower stimuli, resulting in a greater degree of change.
However, the CCM does not account for dilation differences for looming and receding stimuli.

An alternative temporal estimation model, the quick response model (QRM; Droit-Volet \& Gil, 2009), can account for direction differences in temporal estimation. Here, the dilation of perceived interval length serves to facilitate faster decision-making. This model is closely aligned with models predicting that increased arousal and attentional capture will elicit interval overestimation. Events that elicit arousal and attentional capture often also require a quick response (DroitVolet \& Meck, 2007; Lejeune, 1998). More controversially, the model suggests that perceived slowing of an event provides an individual with more subjective time in which to engage an effective behavioral response (Meck, 1996). Movement is an important cue for agency (Abrams \& Christ, 2005; Czeisler et al., 1999): A moving object is likely to be alive and often requires an immediate response from the observer. Furthermore, looming stimuli would be overestimated to a greater extent than receding stimuli, because only approaching forces an observer to prepare a fast and appropriate response for interaction. In the QRM, the overestimation of moving, and especially of looming, stimuli would increase the efficiency of decision-making through attention and arousal when faced with potential threat.

To date, little research has addressed how the speed of expansion/contraction influences and interacts with perceived interval length. In studies investigating the effect of looming and receding motion, the stimuli expanded or contracted to the same degree, regardless of the test interval length. This ensured that the degree of expansion or contraction could not be used as a proxy for elapsed time. However, by keeping the degree of expansion or contraction constant, stimuli expanding or contracting over a shorter interval necessarily change size at a quicker rate than do stimuli that expand or contract over a longer interval. Since longer stimuli are also slower, it is difficult to determine whether the speed of expansion or contraction influences the perception of interval length.

In addition, studies have not investigated whether the dimensionality of the stimuli and of the movement plane used to simulate looming and receding motion has an effect on perceived interval length. In the experiments cited above, looming and receding motion was represented using 2-D discs that expanded/contracted in size in the picture plane. However, expansion/contraction of discs in the picture plane may be perceived differently from that of depth-rendered balls in the depth plane (3-D). Stimuli that appear to move in depth may possess more ecological validity and may more effectively mimic real motion (Abrams \& Christ, 2005). Abrams and Christ found that receding stimuli possessing stereoscopic cues correlated with response speed, but that receding 2-D stimuli did not. An open question is whether the loom/recede distinction for the perception of time may 
disappear with the addition of depth cues indicative of motion through the depth plane or third dimension.

In this study, we investigated whether the variation of contextual cues - speed, movement direction, and depthinfluences and interacts with the subjective perception of interval. We modified a paradigm developed by Wittmann et al. (2010) in which participants compared the duration of a test stimulus with the durations of a series of standard stimuli. We expected to replicate the findings of Wittmann et al. for 2-D stimuli- that is, looming stimuli would be perceived as being longer than receding stimuli of similar durations. However, in addition to the direction of motion, we predicted that the speed of implied movement and the dimensionality (i.e., simulated movement in the picture plane vs. the depth plane) of the stimuli would also influence subjective interval durations and would interact with the direction of motion.

\section{Method}

\section{Participants}

A group of 19 undergraduates (nine males, ten females; mean age $=19.4$ years) participated for course credit in lower-level psychology courses.

\section{Stimuli}

Standard and 2-D stimuli were constructed so as to follow Wittmann et al. (2010). In all conditions, the standardduration stimuli were represented using a solid black circle $3.5 \mathrm{~cm}$ in diameter. Stationary test stimuli were represented using a solid yellow circle with a $3.5-\mathrm{cm}$ diameter. Pictureplane (2-D) looming motion was simulated by presenting a series of solid yellow circles with successively larger diameters. However, to keep the speed of stimulus motion constant within a block, the degree to which the circles' diameters expanded depended on the length of the test interval. For the longest of the five test intervals in a given block, the diameter of the circle expanded from 2 to $5 \mathrm{~cm}$. For the shortest test intervals in a given block, the diameters expanded from 2.6 to $4 \mathrm{~cm}$. Receding stimuli were identical to the looming stimuli, except that the order of presentation was reversed.

Depth-plane (3-D) looming motion was simulated by presenting a series of solid yellow depth-rendered balls with successively larger diameters. The extent of diameter expansion was identical to the extent for 2-D looming stimuli. For looming stimuli, as the ball expanded in size, the center of the solid yellow ball moved along a linear perspective trajectory in order to mimic stereoscopic cues indicative of 3-D motion. Receding stimuli were identical to the 3-D looming stimuli, except that the order of presentation was reversed.

\section{Procedure}

The procedure was modeled after that of Wittmann et al. (2010). Figure 1 illustrates the stimuli and the procedure. Participants were tested individually and sat approximately $60 \mathrm{~cm}$ in front of a 17-in. monitor. Each trial consisted of five images (Fig. 1). The first, second, third, and fifth images were stationary black stimuli presented for a standard duration. As in New and Scholl (2009), the standard duration was $900 \mathrm{~ms}$ in the fast blocks and $1,350 \mathrm{~ms}$ in the slow blocks. The fourth image was always a yellow stimulus presented for a test duration. The interval in-between the stimuli varied randomly from 1,000 to $2,000 \mathrm{~ms}$ in order to reduce temporal markers. After the fifth image, participants pressed the "l" key on the keyboard to indicate that they had perceived the test stimulus duration to be longer than the standard stimulus duration, or the "s" key if they had perceived the standard stimulus to be shorter than the test stimulus.

Each participant completed four blocks of trials presented in a random order: 2-D fast, 2-D slow, 3-D fast, and 3-D slow. Within each block, the test stimuli were presented in the three different movement direction conditions-loom, recede, and stationary - at five different intervals. The fastblock intervals were $600,700,800,900$, and $1,000 \mathrm{~ms}$, and

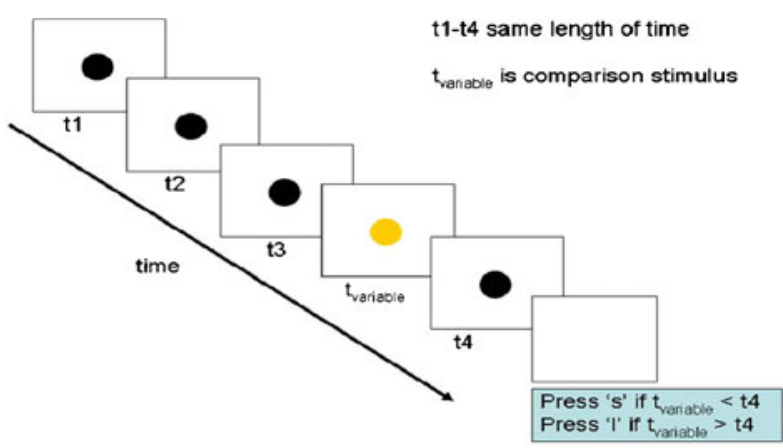

2D Stimulus:

3D Stimulus:

Fig. 1 Stimuli and experimental procedure. Participants viewed three successive black stimuli of a standard duration, followed by a yellow test stimulus and a final black standard-duration stimulus. The participants then indicated whether the perceived duration of the test stimulus was longer or shorter than the test stimuli. The 2-D stimuli were animations of expanding or contracting discs in the picture plane. The "3-D" stimuli were animations of depth-rendered balls expanding or contracting along linear perspective lines, in order to mimic motion in the depth plane 
the slow-block intervals were $900,1,050,1,200,1,350$, and $1,500 \mathrm{~ms}$. For both the fast and slow blocks, three of the test durations were shorter than the standard duration, one was equal to the standard duration, and one was longer than the test duration. The test durations were shorter, on average, than the standard durations because test stimuli are often overestimated (New \& Scholl, 2009). Although this may have affected the distribution of responses, the effect would be constant across conditions.

The fast and slow blocks differed in the speeds of presentation of the same image sequence; the average interval length in the fast blocks was $50 \%$ shorter than in the slow blocks. Thus, the expansion/contraction of looming and receding stimuli simulated in the fast blocks appeared to be $50 \%$ faster than in the slow blocks. Each trial condition was repeated twice within a block, and the trials were presented randomly. In total, 30 trials were presented per block, for a total of 120 trials overall. The entire experiment took $35 \mathrm{~min}$ to complete.

\section{Results}

For each participant and condition, the proportion of "long" responses was calculated. Figure 2 illustrates the mean proportions of "long" responses across test durations for each condition. Psychometric functions were fit for each participant (see Mate et al., 2009). Logit transformation was applied on the proportions of "long" answers $(p)$, so that $\operatorname{logit}(p)=\ln [p /(1-p)]=\alpha+\beta \mathrm{X}$, where $\mathrm{X}$ is the duration of the test stimulus. The point of subjective equality (PSE) defined by the value of the stimulus for a probability of .5 was also estimated $(\mathrm{PSE}=-\beta / \alpha)$. The PSE value represents the duration of the test stimulus that the participant perceives as being equal to the duration of the standard stimulus. Two participants' data could not be adequately modeled and were removed from subsequent analyses.

To be able to compare the fast and slow speed conditions, we calculated difference scores between each PSE and the relevant standard value, with positive values representing temporal overestimation. We submitted these difference scores to a repeated measures analysis of variance (ANOVA) with the factors Dimensionality (2-D, 3D), Speed (slow, fast), and Direction (loom, recede, stationary) (Fig. 3). Significant main effects were found for speed $\left[F(1,16)=26.26, p<.0001, \eta^{2}=.99\right]$ and direction $\left[F(2,32)=16.69, p<.0001, \eta^{2}=.98\right]$, but not for dimensionality $\left[F(1,16)<1, \eta^{2}=.06\right]$. Faster stimuli (mean $=0.18, S E=0.02)$ were overestimated to a greater extent than slower stimuli (mean $=0.06, S E=0.02$ ), and moving stimuli were overestimated to a greater extent than stationary stimuli. Specifically, post-hoc comparisons

\section{2-D Fast}

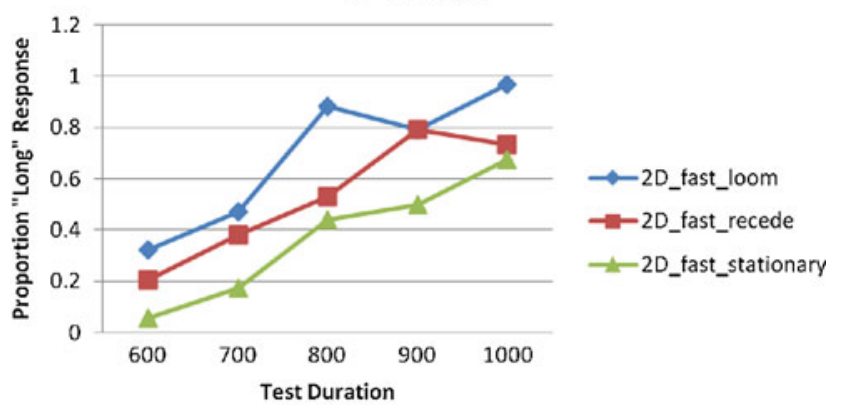

3-D Fast

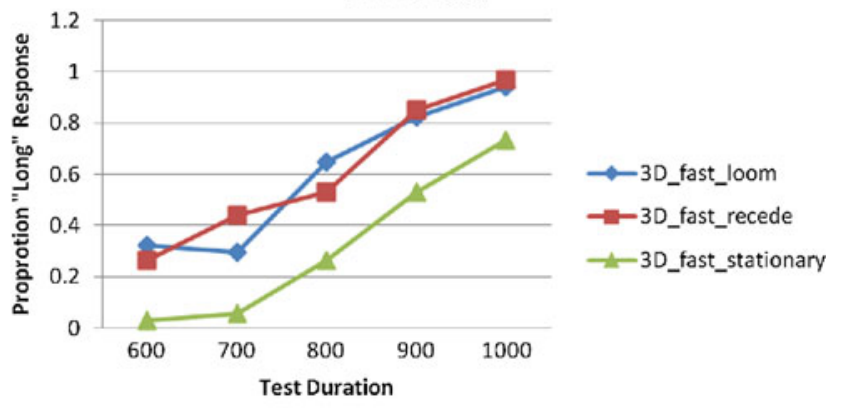

2-D Slow

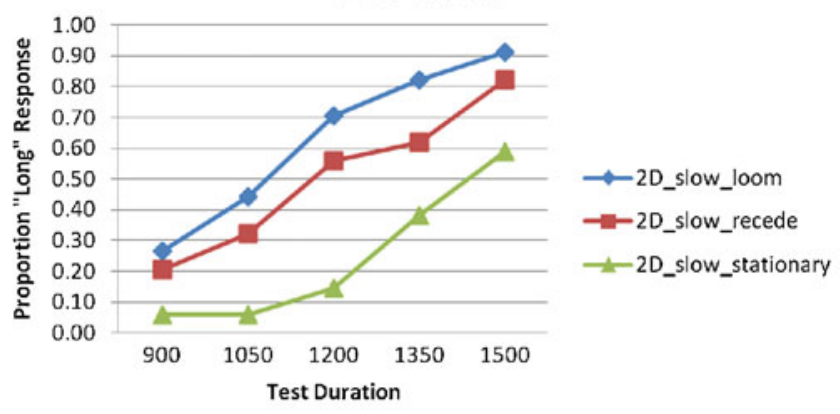

3-D Slow

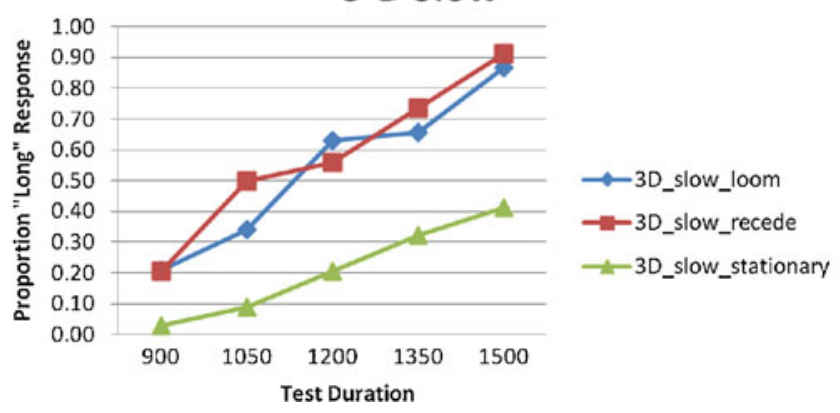

Fig. 2 Psychometric functions for fast and slow 2-D and depthrendered " 3 -D" stimuli in the loom, recede, and stationary conditions. Mean proportions of "long" responses are plotted across test stimulus durations

showed significant differences between stationary and looming [2-D slow, $t(1,16)=4.32, p=.001 ; 3$-D slow, $t(1,16)=5.49, p<.0001 ; 3$-D fast, $t(1,16)=3.79, p=$ .002 ] as well as receding [2-D slow, $t(1,16)=2.72, p=$ 


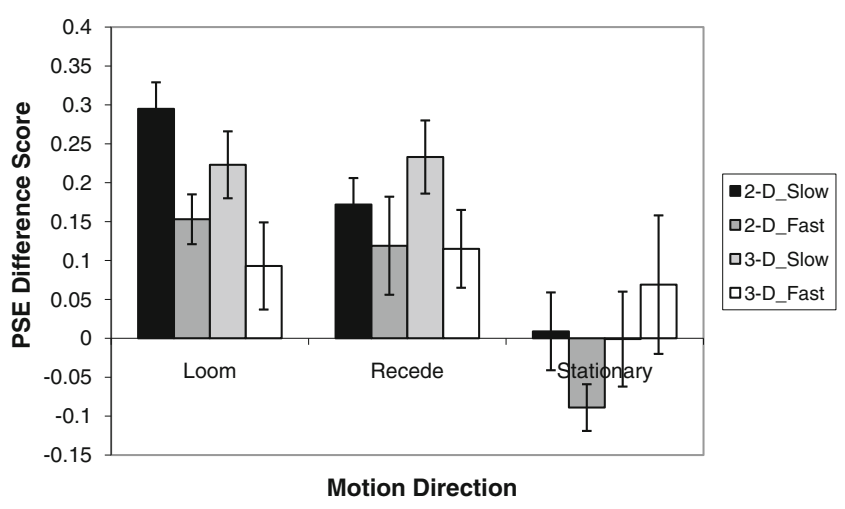

Fig. 3 Difference scores (i.e., standard duration - PSE) for fast and slow 2-D and depth-rendered " 3 -D" stimuli in the loom, recede, and stationary conditions. Positive values indicate overestimation

.015 ; 3-D slow, $t(1,16)=4.12, p<.001 ; 3$-D fast, $t(1,16)$ $=3.15, p=.006]$ stimuli, with the exception of the 2-D fast recede condition $[t(1,16)=1.64, p=.164]$.

Notably, a significant Dimensionality $\times$ Direction interaction was observed $\left[F(2,32)=4.29, p=.022, \eta^{2}=.71\right]$ : 2-D but not 3-D stimuli showed significant differences between the loom and recede conditions. Post-hoc analyses of the 2-D stimuli confirmed a significant loom versus recede difference $\left[F(1,16)=5.76, p=.029, \eta^{2}=.61\right]$, indicating that looming stimuli (mean $=0.21, S E=0.03$ ) elicited greater overestimation than did receding stimuli (mean $=0.17, S E=0.03$ ). However, 3-D stimuli did not show a loom $($ mean $=0.20, S E=0.03)$ versus recede $($ mean $=0.22, S E=0.04)$ direction distinction $[F(1,16)$ $\left.<1, \eta^{2}=.07\right]$.

Finally, we conducted post-hoc comparisons to examine whether the degrees of overestimation differed for 2-D versus 3-D stimuli for the different movement directions. We found no difference in temporal estimation between 2-D and 3-D stationary stimuli [fast, $t(1,16)=2.08, p=$ .06 ; slow, $t(1,16)=-1.16, p=.28]$, indicating similar processing for discs and depth-rendered balls. The 2-D stimuli did elicit greater overestimation than 3 -D stimuli for fast-moving looming stimuli [fast, $t(1,16)=2.42, p=$ $.028]$, but not for slow-moving stimuli $[t(1,16)=-1.16$, $p=.27]$. In contrast, 3-D stimuli tended to produce greater overestimation than 2-D stimuli for receding stimuli, but this difference was only significant for the slow-moving stimuli [fast, $t(1,16)=1.04, p=.312$; slow, $t(1,16)=$ 2.42, $p=.028]$.

A Dimensionality $\times$ Speed interaction was also observed $\left[F(1,16)=6.95, p=.018, \eta^{2}=0.70\right]$. Speed had a greater effect for 2-D stimuli (fast $=0.21, S E=0.03$; slow $=0.02, S E=0.03$ ) than for 3-D stimuli (fast $=0.15$, $S E=0.03$; slow $=0.10, S E=0.03)$. The Speed $\times$ Direction $\left[F(2,32)<1, \eta^{2}=.11\right]$ and three-way $[F(2,32)=1.09$, $\left.p=.40, \eta^{2}=.22\right]$ interactions were not significant.

\section{Discussion}

The results of this study add to a growing body of research indicating that specific contextual factors influence our perception of time. Specifically, we examined the influence and interaction of stimulus/movement dimensionality, movement direction, and movement speed on estimates of interval duration. As a novel result of this study, we established the interaction of stimulus dimensionality with movement direction and speed. Using a paradigm developed by Wittmann et al. (2010), we compared estimates of temporal duration using two-dimensional stimuli with picture-planesimulated movement (2-D) and using depth-rendered stimuli with depth-plane-simulated movement (3-D). Consistent with previous studies, we found an influence of simulated motion on the perception of time. The durations of moving stimuli were overestimated as compared to those of stationary stimuli. Consistent with previous research (e.g., Brown, 1995), participants were more likely to indicate that the test stimulus duration was longer than the standard duration (i.e., temporal overestimation) if the test stimuli simulated motion than if the test stimuli remained stationary.

Speed of movement also influenced perceived interval duration: Stimuli that expanded or contracted at a faster rate elicited a greater degree of overestimation when compared with similar stimuli that moved at a slower speed. However, speed also interacted with stimulus dimensionality. For the fast speed, 2-D stimuli were overestimated more than 3-D stimuli, especially for the loom direction, whereas for the slow speed, 3-D stimuli were overestimated more than 2-D stimuli, especially in the recede direction. Since the fast and slow stimuli moved comparable distances, the CCM would have difficulty explaining the overestimation of faster-moving stimuli. Conversely, speed differences can be explained by the QRM, as a fast-moving object requires a quicker response than does a slow-moving object, but this model would have more difficulty explaining the interaction.

Nonetheless, our most interesting finding was an interaction between movement and stimulus dimensionality. Previous research had shown that looming stimuli elicited dilation in perceived interval length but that receding stimuli did not (New \& Scholl, 2009; van Wassenhove et al., 2008; Wittmann et al., 2010). However, this previous research only simulated motion using 2-D stimuli. In our study, simulated depth-plane motion (nonstereoscopic 3-D) elicited dilation in perceived interval length in both the loom and recede conditions, but this loom/recede distinction for the perception of time only occurred for $2-\mathrm{D}$, and not for 3-D, simulated motion.

One explanation for the loom/recede difference between 2-D and 3-D stimuli relates to the perception of 2-D receding motion. In this study, the 2-D receding stimuli were contracting discs whose centers did not change location. 
An interpretation of the differing temporal perceptions of 2-D looming and receding stimuli is that only 2-D looming stimuli, and not the 2-D receding stimuli, were perceived as moving; it is possible that the observed difference in the literature between 2-D looming and receding stimuli may result from 2-D receding stimuli being perceived as stationary and 2-D looming stimuli being perceived as moving. Since motion tends to universally elicit overestimation, the overestimation of 2-D looming stimuli observed in previous studies could merely be an artifact of simulating motion in 2-D.

If this is the case, and 2-D receding objects are not actually perceived as moving, the CCM can adequately account for the difference between looming and receding motion. In the context of CCM, if 2-D receding objects are not perceived as moving, they would possess a lower total number of changes as compared to 2-D looming objects, producing a shorter perceived duration. However, comparisons of time estimation between receding and stationary stimuli suggest that receding motion is perceived to some extent in slower-moving, if not in faster-moving, 2-D stimuli.

Also, these results appear to contradict the QRM model. One would expect that a 3-D looming object would capture as much (if not more) attention, be as arousing, and require an equally urgent response as a 2-D looming object, because 3-D images possess more ecological validity than do 2-D images. Nonetheless, the present results can be reconciled with the QRM. In our ancestral context, human beings were not uniformly prey, but also frequently predators (Abrams \& Christ, 2005). A receding object might be indicative of a fleeing animal, and a slowed perception of time when presented with a receding object might aid in effective hunting. This hypothesis could be tested in future experiments using objects that not only move toward and away from participants, but also objects that move to the left and right. Given that movement generally represents an important cue of agency, it would be reasonable to assume that the body should respond to all types of motion. Since the consequences for not responding to a potentially threatening animal are greater than the repercussions associated with responding when no threat exists, evolutionary theory would argue that humans should possess a response bias (Öhman, 1997). In conclusion, this study has demonstrated that time perception can be influenced by a variety of interacting contextual cues, and that models of temporal estimation need to provide mechanisms to account for changing contextual conditions.

\section{References}

Abrams, R. A., \& Christ, S. E. (2005). The onset of receding motion captures attention: Comment on Franconeri and Simons (2003). Perception \& Psychophysics, 67, 219-223. doi:10.3758/ BF03206486
Angrilli, A., Cherubini, A., \& Manfredini, S. (1997). The influence of affective factors on time perception. Perception \& Psychophysics, 59, 972-982.

Block, R. A. (1982). Temporal judgments and contextual change. Journal of Experimental Psychology: Learning, Memory, and Cognition, 8, 530-544.

Block, R. A., \& Zakay, D. (1997). Prospective and retrospective duration judgments: A meta-analytic review. Psychonomic Bulletin \& Review, 4, 184-197. doi:10.3758/BF03209393

Brown, S. W. (1995). Time, change, and motion: The effects of stimulus movement on temporal perception. Perception \& Psychophysics, 57, 105-116. doi:10.3758/BF03211853

Brown, S. W. (1997). Attentional resources in timing: Interference effects in concurrent temporal and nontemporal working memory tasks. Perception \& Psychophysics, 59, 1118-1140. doi:10.3758/ BF03205526

Buhusi, C., \& Meck, H. (2002). Differential effects of methamphetamine and haloperidol on the control of an internal clock. Behavioral Neuroscience, 116, 291-297.

Burle, B., \& Casini, L. (2001). Dissociation between activation and attention effects in time estimation: Implications for the internal clock models. Journal of Experimental Psychology: Human Perception and Performance, 27, 195-205.

Czeisler, C. A., Duffy, J. F., Shanahan, T. L., Brown, E. N., Mitchell, J. F., Rimmer, D. W., \& Kronauer, R. E. (1999). Stability, precision, and near-24-hour period of the human circadian pacemaker. Science, 284, 2177-2181. doi:10.1126/science.284.5423.2177

Droit-Volet, S. (2003). Alerting attention and time perception in children. Journal of Experimental Child Psychology, 85, 372-384.

Droit-Volet, S., \& Gil, S. (2009). The time-emotion paradox. Philosophic Transactions of the Royal Society, 364, 1943-1953.

Droit-Volet, S., \& Meck, W. H. (2007). How emotions colour our perception of time. Trends in Cognitive Sciences, 11, 504-513. doi:10.1016/j.tics.2007.09.008

Droit-Volet, S., \& Wearden, J. (2002). Speeding up an internal clock in children? Effects of visual flicker on subjective duration. Quarterly Journal of Experimental Psychology, 55, 193-211.

Eagleman, D. M., \& Pariyadath, V. (2009). Is subjective duration a signature of coding efficiency? Philosophical Transactions of the Royal Society B, 364, 1841-1851. doi:10.1098/rstb.2009.0026

Fraisse, P. (1984). Perception and estimation of time. Annual Review of Psychology, 35, 1-37. doi:10.1146/annurev.ps.35.020184.000245

Franconeri, S. L., Hollingworth, A., \& Simons, D. J. (2005). Do new objects capture attention? Psychological Science, 16, 275-281. doi:10.1111/j.0956-7976.2005.01528.x

Kanai, R, Paffen, C. L. E., Hogendoorn, H., \& Verstraten, F. A. J. (2006). Time dilation in dynamic visual display. Journal of Vision, 6(12), 8:1421-1430. doi:10.1167/6.12.8

Lejeune, H. (1998). Switching or gating? The attentional challenge in cognitive models of psychological time. Behavioral Processes, 44, 127-145.

Lewis, P. A., \& Miall, R. C. (2003). Brain activation patterns during measurement of sub- and supra-second intervals. Neuropsychologia, 41, 1583-1592.

Mate, J., Pires, A. C., Campoy, G., \& Estaun, S. (2009). Estimating the duration of visual stimuli in motion environments. Psicológica, 30, 2878-300.

Meck, W. H. (1996). Neuropharmacology of timing and time perception. Cognitive Brain Research, 3, 227-242.

New, J. J., \& Scholl, B. J. (2009). Subjective time dilation: Spatially local, object-based, or a global visual experience? Journal of Vision, 9(2), 4:1-11. doi:10.1167/9.2.4

Northoff, G., Heinzel, A., de Greck, M., Bermpohl, F., Dobrowolny, H., \& Panksepp, J. (2006). Self-referential processing in our brain -A meta-analysis of imaging studies on the self. NeuroImage, 31, 440-457. doi:10.1016/j.neuroimage.2005.12.002 
Öhman, A. (1997). As fast as the blink of an eye: Evolutionary preparedness for preattentive processing of threat. In P. J. Lang, R. F. Simons, \& M. T. Balaban (Eds.), Attention and Orienting: Sensory and Motivational Processes (pp. 165-184). Mahwah, NJ: Erlbaum.

Ornstein, R. E. (1969). On the experience of time. Harmondsworth, U.K.: Penguin.

Ortega, L., Lopez, F., \& Church, R. M. (2009). Modality and intermittency effects on time estimation. Behavioral Processes, 81, 270 273. doi:10.1016/j.beproc.2009.02.009

Penney, T., Holder, M., \& Meck, W. H. (1996). Clonidine-induced antagonism of norepinephrine modulates the attentional processes involved in peak-interval timing. Experimental Clinical Psychopharmacology, 4, 82-92.

Poynter, W. D. (1989). Judging the duration of time intervals: A process of remembering segments of experience. In I. Levin \& D. Zakay (Eds.), Time and human cognition: A life-span perspective (pp. 305-321). Amsterdam, The Netherlands: Elsevier.

Roelofs, C. O., \& Zeeman, W. P. C. (1951-1952). Influence of different sequences of optical stimuli on the estimation of duration of a given interval of time. Acta Psychologica, 8, 89-128. doi:10.1016/0001-6918(51)90007-8

Schiff, W., Caviness, J. A., and Gibson, J. J. (1962). Persistent fear responses in rhesus monkeys to the optical stimulus of "looming". Science 136, 982-983.
Tayama, T., Nakamura, M., \& Aiba, T. S. (1987). Estimated duration for rotating-spot-pattern. Japanese Psychological Research, 29, $173-183$.

Tse, P. U., Intriligator, J., Rivest, J., \& Cavanagh, P. (2004). Attention and the subjective expansion of time. Perception \& Psychophysics, 66, 1171-1189. doi:10.3758/BF03196844

van Wassenhove, V., Buonomano, D. V., Shimojo, S., \& Shams, L. (2008). Distortions of subjective time perception within and across senses. PLoS ONE, 3, 1437-1450.

Wearden, J. H., \& Penton-Voak, I. (1995). Feeling the heat: Body temperature and the rate of subjective time, revisited. Quarterly Journal Experimental Psychology, 48, 129-141.

Wittmann, M., van Wassenhove, V., Craig, A. D., \& Paulus, M. P. (2010). The neural substrates of subjective time dilation. Frontiers in Human Neuroscience, 4, 2:1-9. doi:10.3389/ neuro.09.002.2010

Yantis, S., \& Egeth, H. E. (1999). On the distinction between visual salience and stimulus-driven attentional capture. Journal of Experimental Psychology: Human Perception and Performance, 25, 661-676. doi:10.1037/0096-1523.25. 3.661

Zakay, D., \& Block, R. A. (2004). Prospective and retrospective duration judgments: An executive-control perspective. Acta Neurobiologiae Experimentalis, 64, 319-328. 